\title{
Analysis, simulation and prediction of contact stresses in articular cartilage of the knee joint
}

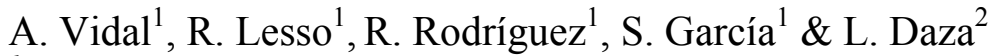 \\ ${ }^{I}$ Department of Mechanical Engineering, \\ Instituto Tecnologico de Celaya, Mexico \\ ${ }^{2} U M A E$ Bajio, IMSS, Mexico
}

\begin{abstract}
Articular cartilage (AC) has an essential function in the best performance of the human body's joints, but it has a limited capacity of regeneration and the initial cause that develops the pathological degenerative process is still unknown. It is believed that cartilage damage due to knee osteoarthritis is mechanically induced. Thus, to investigate such a phenomenon and analyze and simulate the biomechanical behavior of the knee joint, a virtual 3D knee prototype was created using a commercial finite element code, which includes the femur, tibia and $\mathrm{AC}$ as a deformable solid model. This paper shows the stress distribution found in $\mathrm{AC}$ in the femur and the tibia. The factors were obtained by applying a load range of 700 to $2800 \mathrm{~N}, 0^{\circ}$ in flexion and different cases with physiological valgus variation and a graphical model of stress prediction to the femur cartilage was created using the stress behavior with the different factors. In general, the results show that different factors like being overweight (load $>700 \mathrm{~N}$ ) and misalignment (valgus variation) could damage the AC because they increase the stress magnitude and it comes into the cyclic damage range (5-10 MPa), which progressively produces articular cartilage damage and enhances the osteoarthritis phenomenon due to mechanical factors.

Keywords: articular cartilage damage, finite element analysis, contact element, biomechanics, knee joint, osteoarthritis, physiological valgus.
\end{abstract}

\section{Introduction}

The knee is one of the most important joints of human body. It allows, with its flexion-extension movement, the displacement of the body. The human knee joint is composed mainly of the femur, tibia, patella, menisci and articular cartilage. 
Some investigations have found that AC has a limited capacity of being repaired by itself and the initial cause that produces the pathological damage process is still unknown.

It is believed, that cartilage damage by osteoarthritis (OA) is mechanically induced. Some traumatic and ostearthritical injuries produce cartilage damage and the erosion of the articular surface, which leads to a total or partial replacement of knee joint to eliminate the pain and restore the mobility. In addition, there are other causes that produce pain due to OA, like nourishment, pathological causes and bad posture habits, but the principal cause is the overweight problem. This produces misalignment of inferior extremities, tibia and femur principally. The evidence shows that the tibio-femoral contact area is generally the one that presents OA, IMSS [1].

Nowadays, the final solution of OA problem is the total replacement of knee joint using a knee prosthesis, but its average usefulness is less than 10 years and its cost due to surgery and prosthesis is expensive.

It has been estimated, that there will be 3.5 millions of Mexican people with pain and radiological modification by knee osteoarthritis to the 2010 year. Thus, is fundamental to investigate the OA phenomenon. The purpose of this study is to investigate the effect of mechanical factors like misalignment and overweight in articular cartilage damage due to OA.

\section{State of the art}

Some investigations have reported different knee analysis. Haut et al. [2] made a 3D finite element analysis of knee joint; they considered the femur, tibia, menisci, cartilage and ligaments. They reported maximum stress values in the tibio-femoral contact area of $2.36 \mathrm{MPa}$ in the lateral and $2.55 \mathrm{MPa}$ in the medial side. They results were obtained applying a load of $800 \mathrm{~N}$. In the other hand, Donzelli et al. [3], made a contact analysis between two cartilage layers using a 2D finite element model and applying different cartilage behaviors. They showed maximum stress values of $0.125 \mathrm{MPa}$ considering the cartilage like an isotropic material and values of $0.225 \mathrm{MPa}$ considering the cartilage as a transversally isotropic material. Their results were obtained applying a load of $75 \mathrm{~N}$. Also, Thambyah et al. [4] reported a set of in vitro results of different investigations using a pressure sensor introduced in the tibio-femoral contact area. Among these results we can find those reported by McKellop et al. [5], Riegger-Krugh et al. [6] and Ihn et al. [7]. All of them made in vitro experimental test in human knee joint and by using an ink pressure sensor, they reported the following values. McKellop et al. [5] applied $2400 \mathrm{~N}$ of load and reported stress values of 4.1 and 4.6 $\mathrm{MPa}$ in the medial and lateral side, respectively. Riegger-Krugh et al. [6] applied $1200 \mathrm{~N}$ of load and reported stress values of 2.5 and $1.9 \mathrm{MPa}$ in the lateral and medial side, respectively. Ihn et al. [7] applied a load of $1960 \mathrm{~N}$ and reported stress values of 3.86 and $2.52 \mathrm{MPa}$ in the lateral and medial side, respectively. Thambyah et al. [4] using a thin film electronic sensor in real time, reported a pressure of $14 \mathrm{MPa}$ in the medial and $0-11 \mathrm{MPa}$ in the lateral side, they applied up to $2400 \mathrm{~N}$. Also, they reported that with a cyclic stress as low as 
5-10 MPa cartilage damage could appear and consequently, it is important to determine the effect of different factors such as misalignment, overweight, age, etc, that could increase the stress and the damage magnitude and produce articular cartilage erosion.

Finite element analysis made previously on knee joint, did not analyze neither the articular cartilage like a solid model directly nor the damage phenomenon of articular cartilage. Thus, unlike other investigations, in the present investigation we present a 3D finite element analysis where femur and tibia bones, and articular cartilage are considered as deformable bodies, and the principal objective is to develop a virtual prototype of knee joint to study the damage phenomenon of cartilage and its different factors that produce and increase it.

\section{Methods}

The geometry details of each one of the components of the knee joint were obtained from Quiroz [8] and Kapandji [9], whereas in the standardized femur program home page [10], the digitized geometry of femur and tibia was obtained. These models were digitized by Brian Greer and Eric L. Wang, of Nevada's University in Reno, USA. Falcovitz et al. [11] found that the articular cartilage thickness in people with $31 \pm 2$ years old is $2.25 \pm 0.18 \mathrm{~mm}$ and a thickness of $2.26 \pm 0.11 \mathrm{~mm}$ in people with $69 \pm 2$ years old. It was measured in medial condile in human corpse. Adam et al. [12] reported that articular cartilage has a regular distributed thickness in the knee joint in people between 62-94 years old and reported the following thickness: $2.0 \mathrm{~mm}$ in patella; $1.9 \mathrm{~mm}$ in femur and $1.9 \mathrm{~mm}$ in tibia. Barbu-Mcinnis et al. [13] reported cartilage thickness in tibia, which measurements were made on 10 humans, 5 male and 5 female with an average age of 44.8 years old, 5 with osteoarthritis and 5 without it. The average thickness on tibia in people without osteoarthritis was $2.224 \mathrm{~mm}$. Thus, in the present study we consider a virtual prototype with an articular cartilage thickness of $2.25 \mathrm{~mm}$ in femur and $2.224 \mathrm{~mm}$ in tibia.

Due to its highly viscoelastic and anisotropic behavior, mechanical properties of articular cartilage have not been completely determined. To model articular cartilage, two models have been used. The first one is only one phase, isotropic, homogeneous and linearly elastic and the second one is a biphasic model, isotropic, homogeneous, linearly elastic and incompressible. Linear elastic model of only one phase is used when a response in a short time or equilibrium long time response is modeled. Nowadays, the majority of investigations that consider the mechanical properties of articular cartilage to stress analysis suppose a linearly elastic behavior, only one phase, isotropic and homogeneous. The mechanical properties that Haut et al. [2], Li et al. [14] and Wei et al. [15] considered were $\mathrm{E}=15 \mathrm{MPa}$ (elastic modulus), $v=0.475$ (Poisson ratio), which were used in the created prototype. The mechanical properties of bones, which are considered here behaving as an orthotropic material and are used to model femur and tibia are reported by Haut et al. [2], they were obtained of a 30 years old male human corpse, $1.70 \mathrm{~m}$ height and an average weight of $70 \mathrm{Kg}$. 
Other possible cause to consider like a failure in $\mathrm{AC}$ is the erosion produced by friction between the joint components, thus it is known that friction coefficient between two normal cartilage layer is extremely low and it is a fifth of the one exist between two ice blocks, like Bruce [16] indicates and because of that, the failure due to friction is not consider in the virtual prototype.

\subsection{CAD Model}

The digitized bones were imported to a CAD software where the cartilage on femur and tibia, was created from bones surfaces with a constant thickness of $2.25 \mathrm{~mm}$ in femur and $2.224 \mathrm{~mm}$ in tibia. Assemble and alignment were made in the same software considering a $0^{\circ}$ angle in flexion and a $178^{\circ}$ angle in physiological valgus, which is the aligned case.

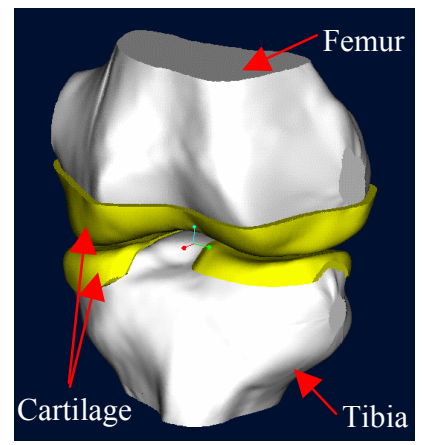

Figure 1: $\quad$ CAD model of knee joint.

\subsection{Finite element model}

The biomechanical simulation of the knee joint was done using commercial finite element software. The contact pairs created were femur-cartilage, cartilage-cartilage and tibia-cartilage, and the necessary parameters to achieve the convergence were established. In the contact components, elements with 8 nodes were used. The contact algorithm used to solve the contact problem was the Augmented Lagrangian Method. This is an iterative series of penalty updates to find the Lagrange multipliers. Compared to the penalty method, the augmented Lagrangian method usually leads to better conditioning and is less sensitive to the magnitude of the contact stiffness coefficient.

The contact pressure $(\mathrm{P})$ is define by:

$$
\mathrm{P}=\left\{\begin{array}{cc}
0 & \text { if } \mu_{\mathrm{n}}>0 \\
\mathrm{~K}_{\mathrm{n}} \mu_{\mathrm{n}}+\lambda_{\mathrm{i}+1} & \text { if } \mu_{\mathrm{n}} \leq 0
\end{array} \text { where, } \quad \lambda_{i=1}=\left\{\begin{array}{c}
\lambda_{i}+K_{n} u_{n} \text { si }\left|u_{n}\right|>\varepsilon \\
\lambda_{i} \quad \text { si }\left|u_{n}\right|<\varepsilon
\end{array},\right.\right.
$$

where, $K_{n}=$ contact normal stiffness, $u_{n}=$ contact gap size, $\varepsilon=$ compatibility tolerance and $\lambda_{\mathrm{i}}=$ Lagrange multiplier component at iteration $\mathrm{i}$. 
The Lagrange multiplier component $\lambda_{\mathrm{i}}$ is computed locally and iteratively.

Due to irregular geometry and material behavior a non-linear analysis was necessary; the finite element used was a tetrahedral with 10 node and 3 dof per node: $\mathrm{x}, \mathrm{y}, \mathrm{z}$ translations. The displacement field of the finite element used is shown in the following equation:

$$
\begin{aligned}
u= & u_{I}\left(2 L_{1}-1\right) L_{1}+u_{J}\left(2 L_{2}-1\right) L_{2}+u_{K}\left(2 L_{3}-1\right) L_{3} \\
& +u_{L}\left(2 L_{4}-1\right) L_{4}+4 u_{M} L_{1} L_{2}+u_{N} L_{2} L_{3}+u_{0} L_{1} L_{3} \\
& +u_{P} L_{1} L_{4}+u_{2} L_{2} L_{4}+u_{R} L_{3} L_{4}
\end{aligned}
$$

with similar expressions for $v$ and $w$, where $\mathrm{L} i$ is the normalized coordinate and $u, v$ and $w$ are displacements vectors along the $\mathrm{x}, \mathrm{y}$ and $\mathrm{z}$ directions, respectively.

\subsection{Boundary conditions}

A $700 \mathrm{~N}$ load was applied to the femur in the $\mathrm{Z}$ negative direction, which is equivalent to the average weight of a person with $70 \mathrm{Kg}$. To simulate the muscles and ligaments effects in the knee joint (which stabilize the joint), the displacement of femur and cartilage in XY plane was constrained and the displacement in $\mathrm{z}$ direction was only possible. The tibia was constrained in all dof on its base simulating that is fixed, it means the joint is in a static equilibrium position.

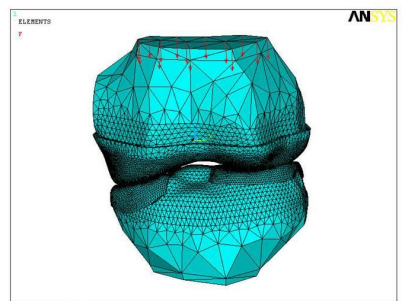

a)

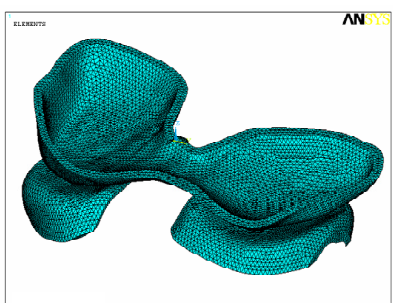

b)

Figure 2: $\quad$ Finite element model a) complete model, b) articular cartilage.

\section{Results analysis}

The stress distribution on the different components of the virtual prototype, considering $0^{\circ}$ in flexion, $178^{\circ}$ in physiological valgus and a load of $700 \mathrm{~N}$, is showed in Figure 3. Results are Von Mises stresses, in MPa.

As shown in Figure 3, the maximum stress is located in the femur-cartilage contact area. Also, the cartilage-cartilage contact area has an equivalent stress magnitude varying from 0 up to $2.4 \mathrm{MPa}$ when applying $700 \mathrm{~N}$ load.

Table 1 compares the result of the present study with other experimental and finite element analyses that have been made. 


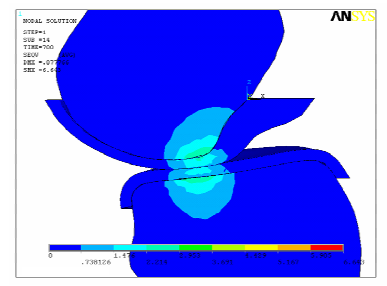

a)

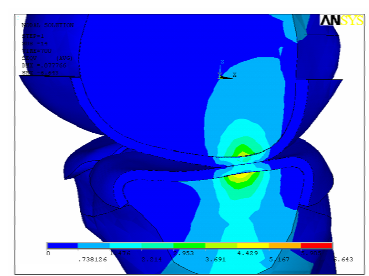

b)

Figure 3: Von Mises stress distribution into knee joint in a sagital cut in the contact area a) lateral side, b) medial side, $\mathrm{MPa}$.

Table 1: Results comparison.

\begin{tabular}{|c|c|c|c|}
\hline Analysis & Method & Applied Load & Contact Stress \\
\hline $\begin{array}{l}\text { McKellop et al. } \\
\text { (1991) }\end{array}$ & $\begin{array}{c}\text { In Vitro analysis } \\
\text { of knee joints } \\
\text { (ink films) }\end{array}$ & $2400 \mathrm{~N}$ & $\begin{array}{l}\text { 4.1 } \mathrm{MPa} \text { (Lateral) } \\
\text { 4.6 MPa (Medial) }\end{array}$ \\
\hline $\begin{array}{c}\text { Donzelli et al. } \\
\text { (1999) }\end{array}$ & $\begin{array}{c}\text { FEA of two cartilage } \\
\text { contact layers }\end{array}$ & $75 \mathrm{~N}$ & $0.225 \mathrm{MPa}$ \\
\hline $\begin{array}{l}\text { Haut et al. } \\
(2002)\end{array}$ & $\begin{array}{c}\text { FEA of knee joint with } \\
\text { ABAQUS }\end{array}$ & $800 \mathrm{~N}$ & $\begin{array}{l}2.36 \mathrm{MPa} \text { (Lateral) } \\
2.55 \mathrm{MPa} \text { (Medial) }\end{array}$ \\
\hline $\begin{array}{l}\text { Thambyah et al. } \\
\text { (2005) }\end{array}$ & $\begin{array}{l}\text { In Vitro analysis } \\
\text { of knee joints } \\
\text { (ink films) }\end{array}$ & $2400 \mathrm{~N}$ & $\begin{array}{l}11 \mathrm{MPa} \text { (Lateral) } \\
14 \mathrm{MPa} \text { (Medial) }\end{array}$ \\
\hline \multirow{3}{*}{ Present analysis } & \multirow{3}{*}{ FEA 3D knee joint } & $75 \mathrm{~N}$ & $\begin{array}{l}0.15-0.59 \mathrm{MPa} \text { (Lateral) } \\
0.20-0.72 \mathrm{MPa} \text { (Medial) }\end{array}$ \\
\hline & & $800 \mathrm{~N}$ & $\begin{array}{l}1.59 \mathrm{MPa} \text { (Lateral) } \\
2.52 \mathrm{MPa} \text { (Medial) }\end{array}$ \\
\hline & & $2400 \mathrm{~N}$ & $\begin{array}{l}\text { 3.01 MPa (Lateral) } \\
\text { 4.32 MPa (Medial) }\end{array}$ \\
\hline
\end{tabular}

The previous table shows that the results obtained in the present analysis, using the virtual prototype are similar to the results of other authors, whose results were obtained using experimental and simulation techniques. With such a correspondence of results, the authors are confident with the present simulation.

\subsection{Physiological valgus variation (misalignment) and load effect}

The virtual prototype was used to analyze the effect of different factors such as misalignament and overweight in the articular cartilage. 19 cases of misalignment, 9 with different varum angles $\left(>178^{\circ}\right)$ and 10 with different valgum angle $\left(<178^{\circ}\right)$, were studied. The applied load was increased up to $2,800 \mathrm{~N}$ because the knee joint reaction forces from walking have been estimated to be as high as three to five times bodyweight according to Morrison [17] and Kuster et al. [18].

Results of misalignment and weight variation show that the contact areas of initial cartilage damage, which are femur-cartilage, cartilage-cartilage and tibiacartilage, are changing with these factors and in general the stress magnitude was increased. 
The results in articular cartilage in femur and tibia showed that the articular cartilage in femur is the one that presents the biggest stress in all the cases under analysis. In some cases, this magnitude is between the damage range of 5 to 10 $\mathrm{MPa}$. Thus, the contact and stress distribution areas for several angles of valgus with an applied load of $2800 \mathrm{~N}$ on femur are shown in Figure 5.
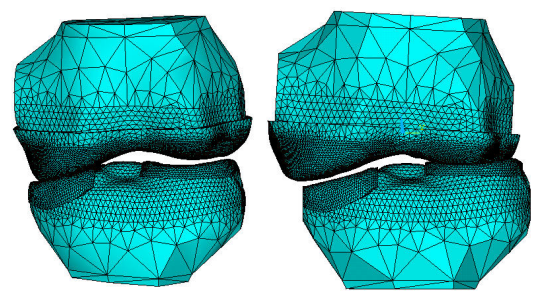

Figure 4: $\quad$ Some cases of misalignment.

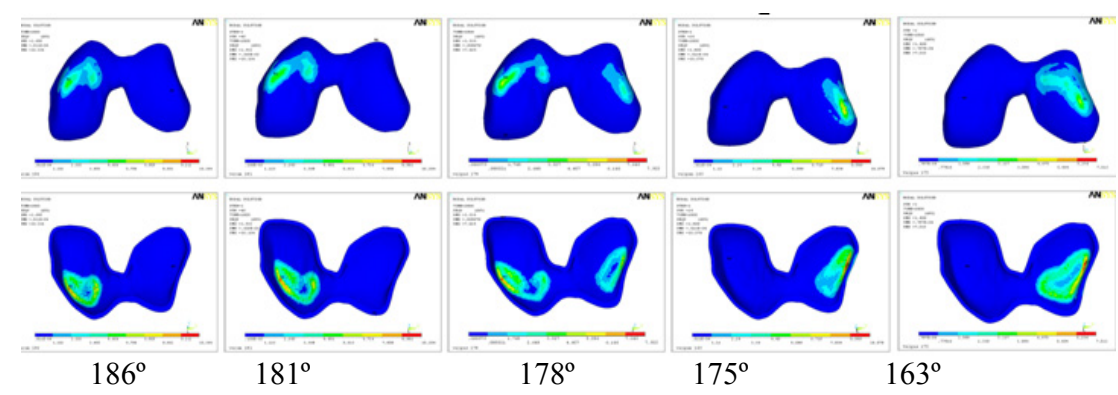

Figure 5: Equivalent stress distribution in articular cartilage in femur at 2800 N. Contact areas for several angles are shown in the figures: external and internal side of cartilage surface, respectively.

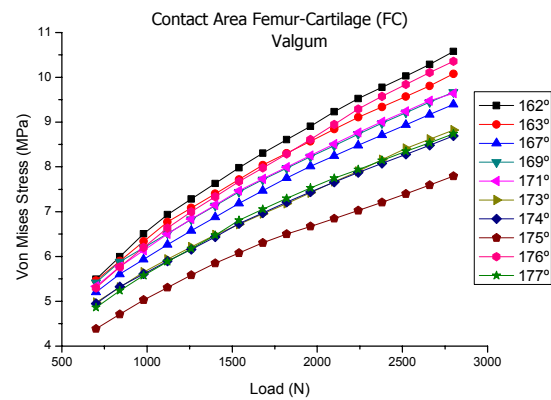

a)

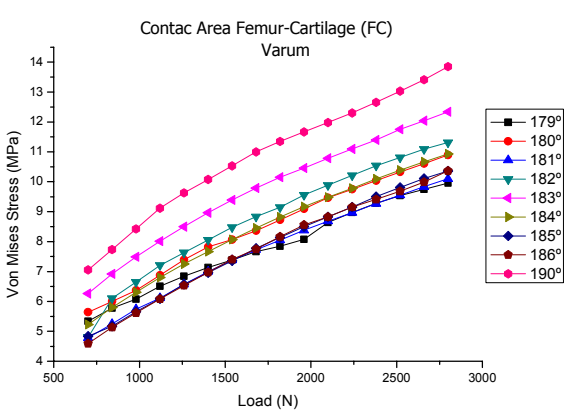

b)

Figure 6: Maximun Von Mises stress behavior in femur cartilage versus applied load. Femur-cartilage contact area in a) valgum case, b) varum case. 
In Figure 6 curves show that the stress in contact area increases when the load rises; however, the increment is linear approximately as the curves shown. Furthermore, the Figure 7 shows that the damage stress magnitude (5-10 MPa) is reached at different load magnitude depending of misalignment grade, but the extreme angles are the cases with the highest misalignment and these correspond to the cases where the damage magnitude is reached with the lowest load.

The non-linear effect of misalignment can be analyzed in Figure 7, where we can see that when the valgus angle diverts of alignment case, the stress magnitude increases in all load cases ( 700 to $2800 \mathrm{~N}$ ). It is important to see that the damage stress magnitude in cartilage-cartilage contact area is reached when a bigger load than $700 \mathrm{~N}$ is transmitted.

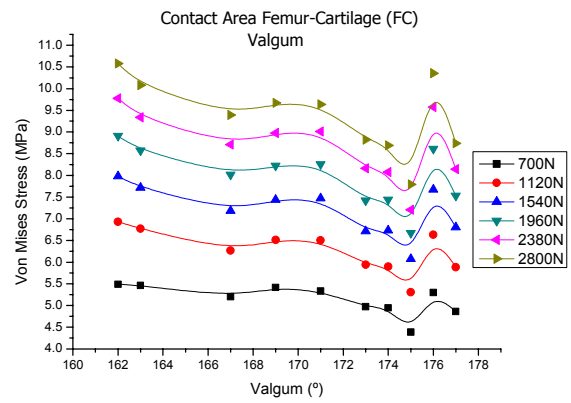

a)

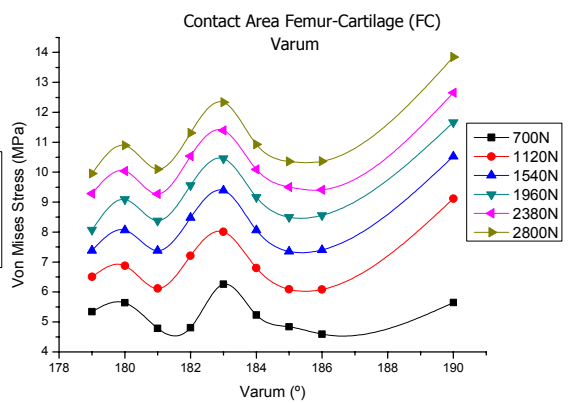

b)

Figure 7: Maximum Von Mises stress behavior in femur cartilage versus valgus angle (misalignment). Femur-cartilage contact area in a) valgum case, b) varum case.

In the other hand, in the femur-cartilage contact area, damage range is reached at lower load than in cartilage-cartilage contact area, having that the majority of the cases of misalignment and overweight fall into the damage range; however, damage magnitude that the factors could produce in articular cartilage depends on stress magnitude with such factors.

\section{Discussion and conclusion}

The biomechanical behavior of articular cartilage is important in order to understand the osteoarthritis phenomenon due to mechanical factors; with the understanding of this phenomenon we could generate alternatives to help people to prevent or diminish the symptoms of this problem.

Thus, instead of doing destructive or invasive test, a computer simulation can be done in advance; this will represent a big success in biomechanics.

The results show that for the aligned case, maximum stress magnitude at $700 \mathrm{~N}$ load is under the damage cyclic range (5-10 MPa) indicated by Thambyah et al. [4], it means that the cartilage makes its normal function, that is to transmit and damp the load at normal conditions. However, results show that different 
factors such as overweight (load $>700 \mathrm{~N})$ and misalignment, could damage the articular cartilage increasing the stress magnitude and the damage magnitude implicitly, and if this magnitude is into the damage cyclic range, it could produce the erosion of articular cartilage progressively.

It is important to say that in the present paper, articular cartilage was considered isotropic, homogeneous and linearly elastic material, taking into account that load is applied in a very short period of time and it has been shown that the fluid within the tissue does not have time to move for short loading times, Thambyah et al. [4]. Despite having done that consideration the obtained results with that material model are too close to those experimentally obtained by other investigations.

It was shown in Figures 6 and 7 that the stress magnitude in articular cartilage depends strongly on the body weight (applied load) and the misalignment (valgus angle), while the damage stress magnitude is reached depending of misalignment and overweight grade.

It is important to indicate that, according to Figure 7, when the valgus angle diverts of aligned case, the stress magnitude is increased in all load range, thus the most stable position with the lowest stress in the joint is the aligned position with the average normal weight of a person.

By using Figures 6 and 7, stress behavior prediction could be done in a graphical way. Thus the different curves shown can be used as prediction graphical models of stress and damage in articular cartilage to different values of load (body weight) and misalignment.

Stresses showed in Figure 6 due to load effect, has a linear behavior approximately; however, stress behavior showed in Figure 7 due to valgus angle (misalignment) is not linear. This non-linear behavior can be attributed to the effect that produces the irregular geometry of the femur condiles (medial and lateral side) and of the tibia (tibial plateau). This irregularity comes from the changes of curvatures ratios of contact surfaces due to variations on alignment.

Finally, the cyclic effect of these factors on the knee joint can produce the articular cartilage damage progressively in femoral condiles, and this enhances the osteoarthritis phenomenon due to mechanical factors.

\section{References}

[1] IMSS (Instituto Mexicano del Seguro Social), León, Guanajuato, México. www.imss.mx

[2] Haut T. L., Hull M. L., Rashid M.M. \& Jacobs C.R. A Finite Element Model of the Human Knee Joint for the Study of Tibio-Femoral Contact. Journal of Biomechanical Engineering, 124, pp. 273-280, 2002.

[3] Donzelli P. S., Spilker R. L., Ateshian G. A. \& Mow V. C. Contact analysis of biphasic transversely isotropic cartilage layers and correlations with tissue failure, Journal of Biomechanics, 32, pp. 1037-1047, 1999.

[4] Thambyah, A., Goh, J.C.H. \& De, S.D. Contact stresses in the knee joint in deep flexion. Medical Engineering \& Physics, 27(4), pp. 329-335, 2005 . 
[5] McKellop H.A., Sigholm G., Redfern F.C., Doyle B., Sarmiento A. \& Luck Sr J.V. The effect of simulated fracture-angulations of the tibia on cartilage pressures in the knee joint. J Bone Joint Surg Am, 73 (9), pp.1382-1391, 1991.

[6] Riegger-Krugh C., Gerhart T.N., Power W.R. \& Hayes W.C. Tibiofemoral contact pressures in degenerative joint disease. Clin Orthop, (348), pp. 233-45, 1998.

[7] Ihn J.C., Kim S.J., Park I.H. In vitro study of contact area and pressure distribution in the human knee after partial and total meniscectomy. Int Orthop, 17(4), pp. 214-218, 1993.

[8] Quiroz G. F., Tratado de Anatomía Humana, Tomo I, pp. 283-292, México 1994.

[9] Kapandji A. I., Fisiología Articular, Tomo II, pp. 74-157. México 1997.

[10] The standardized femur program home page www.tecno.ior.it/VRLAB/researchers/repository/BEL_repository.html

[11] Falcovitz, Y. H., Chan, S. S., Maroudas, A. \& Sah, R L. Compressive properties of normal human articular cartilage: age, depth and compositional dependencies. 47th Annual Meeting, Orthopedic Research Society, February 25-28, 2001, San Francisco, California.

[12] Adam C., Eckstein F., Milz S., Schulte E., Becker C., \& Putz R. The distribution of cartilage thickness in the knee-joints of old-aged individuals - measurement by A-mode ultrasound. Clinical Biomechanics, 13(1), pp. 1-10, 1998 .

[13] Barbu-Mcinnis M., Tamez-Pena J. G. \& Totterman S. Focal Cartilage defect progression detection: measurement of precision and variation in natural characteristics of cartilage thickness maps derived from 3D MRI data. VirtualScopics, LLC, Rochester, NY.

[14] Li G., Gil J., Kanamori A. \& Woo S.L. A Validated Three-Dimensional Computational Model of a Human Knee Joint. Journal of Biomechanical Engineering, 121, pp. 657-662, 1999.

[15] Wei, H.W., Sun, S.S., Jao, S.H.E., Yeh, C.R. \& Cheng, C.K. The influence of mechanical properties of subchondral plate, femur and neck on dynamic stress distribution of the articular cartilage. Medical Engineering \&Physics. 27(4), pp. 295-304, 2005.

[16] Bruce S. R., Transtornos y lesiones del sistema músculo esquelético, Ed. MASSON, pp. 20-27, 40-47,57-61,280-285. México 2000.

[17] Morrison J.B. The mechanics of the knee joint in relation to normal walking. Journal of Biomechanical Engineering, 3, pp. 51-61, 1970.

[18] Kuster M.S., Graeme W.A., Stachowiak G.W. \& Gachter A. Joint load considerations in total knee replacement. J Bone Joint Surg, 79-B(1), pp. 109-113, 1997. 